\title{
Two-loop helicity amplitudes for the production of two off-shell electroweak bosons in gluon fusion
}

\author{
Fabrizio Caola, ${ }^{a}$ Johannes M. Henn, ${ }^{b}$ Kirill Melnikov, ${ }^{c}$ Alexander V. Smirnov ${ }^{d}$ \\ and Vladimir A. Smirnov ${ }^{e}$ \\ ${ }^{a}$ PH Department, TH Unit, CERN, \\ 1211 Geneva 23, Switzerland \\ ${ }^{b}$ Institute for Advanced Study, \\ Princeton, NJ 08540, U.S.A. \\ ${ }^{c}$ Institute for Theoretical Particle Physics, Karlsruhe Institute of Technology, \\ Karlsruhe, Germany \\ ${ }^{d}$ Research Computing Center, Moscow State University, \\ 119991, Moscow, Russia \\ e Skobeltsyn Institute of Nuclear Physics, Moscow State University, 119991 Moscow, Russia \\ E-mail: fabrizio.caola@cern.ch, jmhenn@ias.edu, \\ kirill.melnikov@kit.edu, asmirnov80@gmail.com, \\ smirnov@theory.sinp.msu.ru
}

ABSTRACT: We compute the part of the two-loop virtual amplitude for the process $g g \rightarrow$ $V_{1} V_{2} \rightarrow\left(l_{1} \bar{l}_{1}^{\prime}\right)\left(l_{2} \bar{l}_{2}^{\prime}\right)$, where $V_{1,2}$ are arbitrary electroweak gauge bosons, that receives contributions from loops of massless quarks. Invariant masses of electroweak bosons are allowed to be different from each other. Our result provides an important ingredient for improving the description of gluon fusion contribution to the production of four-lepton final states at the LHC.

KeYwords: QCD Phenomenology, NLO Computations

ARXIV EPRINT: 1503.08759 


\section{Contents}

1 Introduction 1

2 The set up of the computation $\quad 2$

$\begin{array}{lll}3 & \text { Calculation of the amplitude } & 7\end{array}$

4 Conclusions $\quad 9$

$\begin{array}{ll}\text { A The relations between various form factors } & 11\end{array}$

\section{Introduction}

Production of vector boson pairs at the LHC is an important process for a variety of reasons. They include interesting checks of the Standard Model, searches for anomalous vector boson couplings and studies of a dominant background to Higgs boson production. Since the vector boson pair production cross-section is currently known to next-to-next-to-leading order (NNLO) in perturbative QCD $[1,2]$, it is tempting to conclude that theoretical understanding of this process is already good enough and further improvements are unnecessary. Unfortunately, such a conclusion would be premature.

Indeed, since production of vector boson pairs occurs predominantly through the annihilation of quark-antiquark pairs, the one-loop gluon fusion matrix element contributes to $p p \rightarrow V_{1} V_{2}$ cross-section at NNLO for the first time. The increase in the $p p \rightarrow V_{1} V_{2}$ total cross-section caused by the gluon fusion contribution is not very large - it is about five percent in case of the $W^{+} W^{-}$final state [2]. However, this gluon fusion contribution is somewhat larger than the theoretical uncertainty in the production cross section, as estimated in recent NNLO QCD computations [1,2]. Therefore, for these uncertainty estimates to be valid, the current calculations of the gluon fusion contribution to $p p \rightarrow V_{1} V_{2}$ must be accurate to $30-50$ percent. As we explain in the next paragraph, it is conceivable that this is not the case. In addition, the relevance of the gluon-fusion contribution strongly depends on the applied cuts and selection criteria. For example, typical cuts used by ATLAS and CMS in Higgs bosons searches increase the fraction of $g g \rightarrow V_{1} V_{2}$ contribution to the background cross-section to $\mathcal{O}(10 \%)$ [3]. With more aggressive cuts, this fraction can increase to an astounding 30\%, as shown for instance in ref. [4]. Good theoretical control of the gluon fusion process becomes very important in this case.

Since the gluon fusion contribution may play an important role in four-lepton production at hadron colliders, it is important to realize that radiative corrections to $g g \rightarrow V_{1} V_{2}$ can naturally be large. Indeed, $g g \rightarrow V_{1} V_{2}$ is a process where an initial state with large color charge annihilates into a colorless final state. NLO QCD radiative corrections computed for similar processes, such as $g g \rightarrow H, g g \rightarrow H H, g g \rightarrow H Z$ and $g g \rightarrow \gamma \gamma[5-8]$ 
turned out to be as large as 50 - 100 percent. Therefore, to fully control the gluon fusion contribution to vector boson pair production, NLO QCD corrections to $g g \rightarrow V_{1} V_{2}$ need to be calculated. The first step in this direction is the calculation of the two-loop (next-toleading order) scattering amplitude $g g \rightarrow V_{1} V_{2}$. Once the two-loop scattering amplitude becomes available, it will be possible to compute the gluon fusion contribution to vector boson pair production cross-section through NLO in perturbative QCD by supplementing it with the real emission contribution $g g \rightarrow V_{1} V_{2}+g$.

The goal of the present paper is to present a calculation of the $g g \rightarrow V_{1} V_{2}$ helicity amplitudes mediated by loops of massless quarks. We choose to work within the massless approximation because in this case all the relevant two-loop integrals are known [9-12] ${ }^{1}$ and because in many cases massless quarks provide significant fraction of the full amplitude. We note, however, that there are phenomenological situations where top quark loops are important contributors to $g g \rightarrow V_{1} V_{2}$ [15]; our calculation is not applicable to those cases since for top quarks the massless approximation is obviously invalid. We note in this regard that a proper treatment of massive quarks in loop-mediated processes is an important and difficult problem whose solution is not known at the moment. We therefore start with the massless quark contribution to the gluon fusion process $g g \rightarrow V_{1} V_{2}$ which, at the very least, will allow an informed estimate of the magnitude of NLO QCD corrections. Our result can then be supplemented with the contribution of the massive top quark loop once it becomes available. First steps in that direction where recently reported in refs. [16].

The remainder of the paper is organized as follows. In section 2 we discuss the setup of the calculation and the parametrization of the $g g \rightarrow V_{1} V_{2}$ amplitude. In section 3 we describe numerical implementation and checks of the calculated amplitudes and present some numerical results. We conclude in section 4 . Finally, we note that many aspects of the calculation that we report here are similar to what we described in ref. [17]; nevertheless we believe that there are essential differences in the calculation of $q \bar{q} \rightarrow V_{1} V_{2}[12,17]$ and $g g \rightarrow$ $V_{1} V_{2}$ amplitudes at two loops to warrant a discussion of the latter in a separate publication.

\section{The set up of the computation}

We consider the process $g\left(p_{1}\right) g\left(p_{2}\right) \rightarrow\left(V_{1}^{*}\left(p_{3}\right) \rightarrow l\left(p_{5}\right) \bar{l}\left(p_{6}\right)\right)\left(V_{2}^{*}\left(p_{4}\right) \rightarrow l\left(p_{7}\right) \bar{l}\left(p_{8}\right)\right)$. We work in the approximation where quarks of the first two generations are massless and quarks of the third generation are neglected. The CKM matrix is taken to be an identity matrix. We write the matrix element as

$$
\mathcal{M}\left(\lambda_{1}, \lambda_{2}, \lambda_{5}, \lambda_{7}\right)=i\left(\frac{g_{W}}{\sqrt{2}}\right)^{4} \delta^{a_{1} a_{2}} \mathcal{D}_{3} \mathcal{D}_{4} C_{l, V_{2}}^{\lambda_{7}} C_{l, V_{1}}^{\lambda_{5}} \epsilon_{3}^{\mu}\left(\lambda_{5}\right) \epsilon_{4}^{\nu}\left(\lambda_{7}\right) C_{V_{1} V_{2}} \mathcal{A}_{\mu \nu}\left(p_{1}^{\lambda_{1}}, p_{2}^{\lambda_{2}} ; p_{3}, p_{4}\right)
$$

where $a_{1,2}$ are the color indices of the incoming gluons, $g_{W}=e / \sin \theta_{W}$ is the $\mathrm{SU}(2)$ weak coupling, $\mathcal{D}_{i}=1 /\left(p_{i}^{2}-m_{V_{i}}^{2}+i m_{V_{i}} \Gamma_{V_{i}}\right)$ is the $V_{i}$-boson propagator, $\lambda_{1,2}, \lambda_{5}, \lambda_{7}$ are helicities of the incoming gluons and outgoing leptons, $C_{l, V_{2}}^{\lambda_{7}} C_{l, V_{1}}^{\lambda_{5}}$ are helicity-dependent couplings of vector bosons to leptons, and $\epsilon_{3,4}$ are matrix elements for leptonic decays of $V_{1}$ and $V_{2}$ that

\footnotetext{
${ }^{1}$ For earlier results in the case of equal-mass vector bosons, see [13, 14].
} 
we will specify shortly. The factor $C_{V_{1} V_{2}}$ involves sums of couplings of virtual fermions to gauge bosons.

We now give these couplings explicitly. The couplings of electroweak vector bosons to external leptons read

$$
C_{\gamma}^{L, R}=-\sqrt{2} Q_{l} \sin \theta_{W}, \quad C_{l, Z}^{L, R}=\frac{1}{\sqrt{2} \cos \theta_{W}}\left(V_{l} \pm A_{l}\right), \quad C_{l W^{+}}^{\lambda}=C_{l W^{-}}^{\lambda}=\delta_{\lambda L} .
$$

Here, $V_{e}=-1 / 2+2 \sin ^{2} \theta_{W}, V_{\nu}=1 / 2, A_{e}=-1 / 2, A_{\nu}=1 / 2$ and $Q_{l}$ is the lepton electric charge in units of the positron charge. The couplings to virtual quarks, where two massless generations are included, are

$$
\begin{aligned}
C_{\gamma \gamma} & =\frac{20 \sin \theta_{W}^{2}}{9}, & C_{Z Z} & = \\
C_{Z \gamma} & =-\frac{2 \sin \theta_{W}}{\cos \theta_{W}}\left(V_{u} Q_{u}+V_{d} Q_{d}\right), & C_{W^{+} W^{-}} & =1,
\end{aligned}
$$

where $Q_{u, d}$ are the electric charges of up and down quarks in units of the positron charge and $V_{u}=1 / 2-4 / 3 \sin ^{2} \theta_{W}, A_{u}=1 / 2, V_{d}=-1 / 2+2 / 3 \sin ^{2} \theta_{W}, A_{d}=-1 / 2$.

A word of caution is required here. As can be seen from eq. (2.3), the amplitude for the gluon fusion into a pair of vector bosons is proportional to sums of squares of vector and axial-vector couplings of these vector bosons to internal fermions. This means that diagrams with two vector currents and diagrams with two axial currents give identical contributions to the amplitude and that diagrams that involve one vector current and one axial current do not contribute at all. These two features have different origins. The first one is the result of the massless approximation and an ensuing relations between matrix elements of vector and axial currents. The second feature is a direct consequence of $C$-parity conservation [18-20], that enforces the cancellation of these axial-vector terms when contributions of all diagrams are summed up. Therefore, the knowledge of the amplitude for vector-like couplings of gauge bosons to quarks is sufficient to reconstruct the gluon fusion amplitude for electroweak gauge bosons whose couplings to fermions are linear combinations of vector and axial-vector couplings. Similar arguments ensure that diagrams with a single vector boson coupled to two gluons through a closed fermion triangle do not contribute to the amplitude. Indeed, the vector part of these diagrams vanishes because of $C$-parity conservation while the axial part cancels among mass-degenerate isospin doublets ${ }^{2}$.

The scattering amplitude in eq. (2.1) stripped of all non-essential couplings reads

$$
\mathcal{A}=\mathcal{A}_{\mu \nu} \epsilon_{3}^{\mu} \epsilon_{4}^{\nu}=\bar{A}_{\mu \nu \alpha \beta} \epsilon_{1, \lambda_{1}}^{\mu} \epsilon_{2, \lambda_{2}}^{\nu} \epsilon_{3}^{\alpha} \epsilon_{4}^{\beta},
$$

where $\epsilon_{1,2}$ are polarization vectors for gluons with definite helicities. As we just explained, we need to compute the amplitude $\mathcal{A}$ for vector-like interactions of massive vector bosons. We need to decompose the amplitude $\mathcal{A}$ into invariant form factors that are independent of polarization vectors. To do this, we choose physical polarizations for gluons and vector bosons,

$$
\epsilon_{1} \cdot p_{1,2}=0, \quad \epsilon_{2} \cdot p_{1,2}=0, \quad \epsilon_{3} \cdot p_{3}=0, \quad \epsilon_{4} \cdot p_{4}=0 .
$$

\footnotetext{
${ }^{2} \mathrm{~A}$ detailed analysis of triangle diagrams can be found in [15], where the case of non mass-degenerate doublets is considered as well.
} 
It follows from eq. (2.5) that we use $p_{1,2}$ as a reference vector for polarization vectors $\epsilon_{2,1}$, respectively.

To reduce the number of independent scalar products, we employ the Sudakov decomposition of the vector boson momenta, $p_{3,4}=\alpha_{3,4} p_{1}+\beta_{3,4} p_{2} \pm p_{\perp}$. The coefficients $\alpha_{3,4}$ and $\beta_{3,4}$ are expressed in terms of Mandelstam invariants

$$
\alpha_{3}=\frac{m_{3}^{2}-u}{s}, \quad \beta_{3}=\frac{m_{3}^{2}-t}{s}, \quad \alpha_{4}=\frac{m_{4}^{2}-t}{s}, \quad \beta_{4}=\frac{m_{4}^{2}-u}{s} .
$$

Since $\epsilon_{3} \cdot p_{3}=0$ and $\epsilon_{4} \cdot p_{4}=0$, we can choose $\epsilon_{3,4} \cdot p_{1}$ and $\epsilon_{3,4} \cdot p_{2}$ as independent scalar products; $\epsilon_{3,4} \cdot p_{\perp}$ are then given by linear combinations of $\epsilon_{3,4} \cdot p_{1,2}$. Given these constraints, the scattering amplitude is represented by the following expression

$$
\begin{aligned}
\mathcal{A}= & T_{1}\left(\epsilon_{1} \cdot \epsilon_{2}\right)\left(\epsilon_{3} \cdot \epsilon_{4}\right)+T_{2}\left(\epsilon_{1} \cdot \epsilon_{3}\right)\left(\epsilon_{2} \cdot \epsilon_{4}\right)+T_{3}\left(\epsilon_{1} \cdot \epsilon_{4}\right)\left(\epsilon_{2} \cdot \epsilon_{3}\right) \\
& +\left(\epsilon_{1} \cdot \epsilon_{2}\right)\left(T_{4}\left(p_{1} \cdot \epsilon_{3}\right)\left(p_{1} \cdot \epsilon_{4}\right)+T_{5}\left(p_{1} \cdot \epsilon_{3}\right)\left(p_{2} \cdot \epsilon_{4}\right)\right. \\
& \left.+T_{6}\left(p_{2} \cdot \epsilon_{3}\right)\left(p_{1} \cdot \epsilon_{4}\right)+T_{7}\left(p_{2} \cdot \epsilon_{3}\right)\left(p_{2} \cdot \epsilon_{4}\right)\right) \\
& +\left(\epsilon_{1} \cdot \epsilon_{3}\right)\left(p_{\perp} \cdot \epsilon_{2}\right)\left(T_{8}\left(p_{1} \cdot \epsilon_{4}\right)+T_{9}\left(p_{2} \cdot \epsilon_{4}\right)\right) \\
& +\left(\epsilon_{1} \cdot \epsilon_{4}\right)\left(p_{\perp} \cdot \epsilon_{2}\right)\left(T_{10}\left(p_{1} \cdot \epsilon_{3}\right)+T_{11}\left(p_{2} \cdot \epsilon_{3}\right)\right) \\
& +\left(\epsilon_{2} \cdot \epsilon_{3}\right)\left(p_{\perp} \cdot \epsilon_{1}\right)\left(T_{12}\left(p_{1} \cdot \epsilon_{4}\right)+T_{13}\left(p_{2} \cdot \epsilon_{4}\right)\right) \\
& +\left(\epsilon_{2} \cdot \epsilon_{4}\right)\left(p_{\perp} \cdot \epsilon_{1}\right)\left(T_{14}\left(p_{1} \cdot \epsilon_{3}\right)+T_{15}\left(p_{2} \cdot \epsilon_{3}\right)\right) \\
& +\left(\epsilon_{1} \cdot p_{\perp}\right)\left(\epsilon_{2} \cdot p_{\perp}\right)\left(T_{17}\left(p_{1} \cdot \epsilon_{3}\right)\left(p_{1} \cdot \epsilon_{4}\right)+T_{18}\left(p_{1} \cdot \epsilon_{3}\right)\left(p_{2} \cdot \epsilon_{4}\right)+T_{19}\left(p_{2} \cdot \epsilon_{3}\right)\left(p_{1} \cdot \epsilon_{4}\right)\right. \\
& \left.+T_{20}\left(p_{2} \cdot \epsilon_{3}\right)\left(p_{2} \cdot \epsilon_{4}\right)\right)+\left(\epsilon_{3} \cdot \epsilon_{4}\right)\left(p_{\perp} \cdot \epsilon_{1}\right)\left(p_{\perp} \cdot \epsilon_{2}\right) T_{16} .
\end{aligned}
$$

The form-factors $T_{1 \ldots 20}$ are functions of Mandelstam kinematic variables $s, t, u$ and invariant masses of the two vector bosons $m_{3,4}$.

The problem with the calculation of the scattering amplitude "as it is" is that carrying around polarization vectors for external gluons and vector bosons is extremely expensive for computations that employ integration-by-parts identities [21, 22], the main vehicle for multi-loop calculations. For this reason, we need a procedure that allows us to compute amplitudes but where no vectors, except for momenta of external particles, appear in the calculation. A suitable method employs projection operators. The idea is to compute the amplitude $\mathcal{A}$ in eq. (2.7) with polarization vectors $\epsilon_{1 \ldots 4}$ substituted by linear combinations of external momenta $p_{1,2,3,4}$. This procedure allows us to compute the "projected" amplitude $\mathcal{A}$ in terms of form factors $T_{1 \ldots 20}$ and also using integration-by-parts identities since polarization vectors completely disappear from the calculation. By choosing a sufficient number of independent "projection operators", we produce a system of equations that we can solve for the form factors $T_{1 \ldots 20}$.

However, there is a subtlety. The expression for the amplitude in eq. (2.7) was written under the assumption that polarization vectors satisfy the transversality conditions eq. (2.5); those conditions will be definitely violated if polarization vectors are replaced by linear combinations of external momenta. To get around this problem, we write the amplitude as

$$
\mathcal{A}=\bar{A}_{\mu \nu \alpha \beta} \mathcal{P}_{12}^{\mu \mu_{1}} \mathcal{P}_{12}^{\nu \nu_{1}} \mathcal{P}_{3}^{\alpha \alpha_{1}} \mathcal{P}_{4}^{\beta \beta_{1}} \epsilon_{1 \mu_{1}} \epsilon_{2 \nu_{1}} \epsilon_{3 \alpha_{1}} \epsilon_{4 \beta_{1}}
$$


where

$$
\mathcal{P}_{12}^{\mu \nu}=-g^{\mu \nu}+\frac{p_{1}^{\mu} p_{2}^{\nu}+p_{1}^{\nu} p_{2}^{\mu}}{p_{1} \cdot p_{2}}, \quad \mathcal{P}_{3}^{\mu \nu}=-g^{\mu \nu}+\frac{p_{3}^{\mu} p_{3}^{\nu}}{p_{3}^{2}}, \quad \mathcal{P}_{4}^{\mu \nu}=-g^{\mu \nu}+\frac{p_{4}^{\mu} p_{4}^{\nu}}{p_{4}^{2}} .
$$

We then note that in eq. (2.8) we can use any vector $\epsilon_{i}$ to calculate the amplitude since projection operators $\mathcal{P}_{12}, \mathcal{P}_{3,4}$ automatically ensure the transversality constraints $p_{1,2} \cdot \epsilon_{1}=$ $0, p_{1,2} \cdot \epsilon_{2}=0, p_{3} \cdot \epsilon_{3}=0$ and $p_{4} \cdot \epsilon_{4}=0$. We can also replace $\epsilon_{1}^{\mu} \epsilon_{2}^{\nu} \epsilon_{3}^{\alpha} \epsilon_{4}^{\beta}$ with an arbitrary rank-four tensor since it will also be projected on the appropriate transverse space.

We now list all projection operators that we use in the computation of $g g \rightarrow V_{1} V_{2}$ amplitude. To this end, we define the amplitude contracted with the projection operators

$$
\mathcal{O}^{\mu_{1} \mu_{2} \mu_{3} \mu_{4}}=\bar{A}_{\nu_{1} \nu_{2} \nu_{3} \nu_{4}} \mathcal{P}_{12}^{\nu_{1} \mu_{1}} \mathcal{P}_{12}^{\nu_{2} \mu_{2}} \mathcal{P}_{3}^{\nu_{3} \mu_{3}} \mathcal{P}_{4}^{\nu_{4} \mu_{4}}
$$

We also define a tensor that is a projector on the vector space that is orthogonal to the collision plane. It reads

$$
t_{\nu}^{\mu}=\delta_{\nu p_{1} p_{2} p_{\perp}}^{\mu p_{1} p_{2} p_{\perp}}
$$

where $\delta_{\nu_{1} p_{1} p_{2} p_{\perp}}^{\mu_{1} p_{1} p_{\perp} p_{\perp}}=\delta_{\nu_{1} \nu_{2} \nu_{3} \nu_{4}}^{\mu_{1} \mu_{2} \mu_{3} \mu_{4}} p_{1}^{\nu_{2}} p_{2}^{\nu_{3}} p_{\perp}^{\nu_{4}} p_{1, \mu_{2}} p_{2, \mu_{3}} p_{\perp, \mu_{4}}$ and

$$
\delta_{\nu_{1} \nu_{2} \nu_{3} \nu_{4}}^{\mu_{1} \mu_{2} \mu_{3} \mu_{4}}=\operatorname{det}\left|g_{\nu_{j \in\{1 \ldots 4\}}}^{\mu_{i \in\{1 \ldots 4}}\right| .
$$

It is clear that contraction of tensor $t_{\mu \nu}$ with any linear combination of $p_{1}, p_{2}$ and $p_{\perp}$ vanishes thanks to the antisymmetry of the determinant in eq. (2.12).

We define twenty projections of the amplitude $\mathcal{A}$ on linear combinations of $T_{1 \ldots 20}$ by making different choices of the "polarization vectors". They are

$$
\begin{aligned}
G_{1} & =\mathcal{O}^{\mu_{1} \mu_{2} \mu_{3} \mu_{4}} g_{\mu_{1} \mu_{2}} g_{\mu_{3} \mu_{4}}, & G_{2} & =\mathcal{O}^{\mu_{1} \mu_{2} \mu_{3} \mu_{4}} g_{\mu_{1} \mu_{3}} g_{\mu_{2} \mu_{4}}, \\
G_{3} & =\mathcal{O}^{\mu_{1} \mu_{2} \mu_{3} \mu_{4}} g_{\mu_{1} \mu_{4}} g_{\mu_{2} \mu_{3}}, & G_{4} & =p_{\perp}^{-4} s^{-2} \mathcal{O}^{p_{\perp} p_{\perp} p_{1} p_{1},}, \\
G_{5} & =p_{\perp}^{-4} s^{-2} \mathcal{O}^{p_{\perp} p_{\perp} p_{1} p_{2}}, & G_{6} & =p_{\perp}^{-4} s^{-2} \mathcal{O}^{p_{\perp} p_{\perp} p_{2} p_{1}}, \\
G_{7} & =p_{\perp}^{-4} s^{-2} \mathcal{O}^{p_{\perp} p_{\perp} p_{2} p_{2}}, & G_{8} & =4 p_{\perp}^{-6} s^{-2} \mathcal{O}^{p_{\perp} p_{\perp} \mu_{3} \mu_{4}} t_{\mu_{3} \mu_{4}}, \\
G_{9} & =4 p_{\perp}^{-6} s^{-6} \mathcal{O}^{\mu_{1} \mu_{2} p_{1} p_{1}} t_{\mu_{1} \mu_{2}}, & G_{10} & =8 p_{\perp}^{-4} s^{-3} \mathcal{O}^{p_{\perp} \mu_{2} \mu_{3} p_{1}} t_{\mu_{2} \mu_{3}}, \\
G_{11} & =4 p_{\perp}^{-6} s^{-3} \mathcal{O}^{p_{\perp} \mu_{2} \mu_{3} p_{\perp}} t_{\mu_{2} \mu_{3}}, & G_{12} & =8 p_{\perp}^{-4} s^{-3} \mathcal{O}^{\mu_{1} p_{\perp} p_{1} \mu_{4}} t_{\mu_{1}, \mu_{4}}, \\
G_{13} & =4 p_{\perp}^{-6} s^{-3} \mathcal{O}^{\mu_{1} p_{\perp} p_{\perp} \mu_{4}} t_{\mu_{1}, \mu_{4}}, & G_{14} & =8 p_{\perp}^{-4} s^{-3} \mathcal{O}^{\mu_{1} p_{\perp} \mu_{3} p_{2}} t_{\mu_{1}, \mu_{3}}, \\
G_{15} & =4 p_{\perp}^{-6} s^{-3} \mathcal{O}^{\mu_{1} p_{\perp} \mu_{3} p_{\perp}} t_{\mu_{1}, \mu_{3}}, & G_{16} & =8 p_{\perp}^{-4} s^{-3} \mathcal{O}^{p_{\perp} \mu_{2} p_{1} \mu_{4}} t_{\mu_{2}, \mu_{4}}, \\
G_{17} & =4 p_{\perp}^{-6} s^{-3} \mathcal{O}^{p_{\perp} \mu_{2} p_{\perp} \mu_{4}} t_{\mu_{2}, \mu_{4}}, & G_{18} & =4 p_{\perp}^{-6} s^{-6} \mathcal{O}^{\mu_{1} \mu_{2} p_{1} p_{2}} t_{\mu_{1} \mu_{2}}, \\
G_{19} & =4 p_{\perp}^{-6} s^{-6} \mathcal{O}^{\mu_{1} \mu_{2} p_{2} p_{1}} t_{\mu_{1} \mu_{2}}, & G_{20} & =4 p_{\perp}^{-6} s^{-6} \mathcal{O}^{\mu_{1} \mu_{2} p_{2} p_{2}} t_{\mu_{1} \mu_{2}} .
\end{aligned}
$$

In these equations, we used a simplified notation for the contraction of the tensor $\mathcal{O}$ with a vector $a, \mathcal{O}^{\mu_{1} \ldots \mu_{\ldots} \ldots \mu_{n}} a_{\mu}=\mathcal{O}^{\mu_{1} \ldots a \ldots \mu_{n}}$. Since $G_{1 \ldots 20}$ only depend on scalar products of external momenta and on scalar products of external momenta and the loop momenta, we can express $G_{1 \ldots 20}$ through known master integrals $[9,10]$ by applying integration-byparts identities $[21,22]$. At the same time, the form factors $T_{1 \ldots 20}$ can be written as linear combinations of $G_{1 \ldots 20}$ in a straightforward way. 
To determine physical amplitude for the process $g\left(p_{1}\right) g\left(p_{2}\right) \rightarrow\left(V^{*}\left(p_{3}\right) \rightarrow\right.$ $\left.l\left(p_{5}\right) \bar{l}\left(p_{6}\right)\right)\left(V^{*}\left(p_{4}\right) \rightarrow l\left(p_{7}\right) \bar{l}\left(p_{8}\right)\right)$ we use spinor-helicity notations. Specifically, for the incoming gluons we choose

$$
\epsilon_{1 L}^{\mu}=-\frac{\left[2 \gamma^{\mu} 1\right\rangle}{\sqrt{2}[21]}, \quad \epsilon_{1 R}^{\mu}=\frac{\left\langle 2 \gamma^{\mu} 1\right]}{\sqrt{2}\langle 21\rangle}, \quad \epsilon_{2 L}^{\mu}=-\frac{\left[1 \gamma^{\mu} 2\right\rangle}{\sqrt{2}[12]}, \quad \epsilon_{2 R}^{\mu}=\frac{\left\langle 1 \gamma^{\mu} 2\right]}{\sqrt{2}\langle 12\rangle} .
$$

Since a complex conjugation of the helicity amplitudes reverses all helicities, we only need to consider two, rather than four, cases of equal and unequal helicities. We choose $L_{1} L_{2}$ and $L_{1} R_{2}$ as polarization states for gluons $g_{1}$ and $g_{2}$, respectively. We leave the polarization vectors of the massive vector bosons unspecified at this point. To proceed further, it is convenient to write tensor products of gluon polarization vectors as follows (see e.g. ref. [4])

$$
\begin{aligned}
\epsilon_{1 L, \mu} \epsilon_{2 L, \nu} & =\frac{\langle 12\rangle}{[12] s}\left(p_{1, \mu} p_{2, \nu}+p_{1, \nu} p_{2, \mu}-g_{\mu, \nu} p_{1} \cdot p_{2}+i \epsilon_{\mu \nu p_{1} p_{2}}\right), \\
\epsilon_{1 L, \mu} \epsilon_{2 R, \nu} & =\frac{\left\langle 1 p_{\perp} 2\right]}{\left[1 p_{\perp} 2\right\rangle p_{\perp}^{2} s^{2}}\left(\frac{p_{\perp, \mu} p_{\perp \nu} s^{2}}{4}+s i \epsilon_{p_{1} p_{2} p_{\perp} \mu} p_{\perp, \nu}-\epsilon_{p_{1} p_{2} p_{\perp} \mu} \epsilon_{p_{1} p_{2} p_{\perp} \nu}+(\mu \leftrightarrow \nu)\right) .
\end{aligned}
$$

The transverse momentum $p_{\perp}^{\mu}$ is introduced just before eq. (2.6). We use eq. (2.15) to express the amplitude $\mathcal{A}$ in eq. (2.7) through nine independent Lorentz structures

$$
\begin{gathered}
\mathcal{A}_{\lambda_{1} \lambda_{2}}=\mathcal{N}_{\lambda_{1} \lambda_{2}}\left[F_{1}^{\lambda_{1} \lambda_{2}}\left(p_{1} \cdot \epsilon_{4}\right)\left(p_{1} \cdot \epsilon_{3}\right)+F_{2}^{\lambda_{1} \lambda_{2}}\left(p_{1} \cdot \epsilon_{4}\right)\left(p_{2} \cdot \epsilon_{3}\right)+F_{3}^{\lambda_{1} \lambda_{2}}\left(p_{1} \cdot \epsilon_{3}\right)\left(p_{2} \cdot \epsilon_{4}\right)\right. \\
\quad+F_{4}^{\lambda_{1} \lambda_{2}}\left(p_{2} \cdot \epsilon_{4}\right)\left(p_{2} \cdot \epsilon_{3}\right)+F_{5}^{\lambda_{1} \lambda_{2}} \epsilon_{4} \cdot \epsilon_{3} \\
+i \epsilon_{\mu \nu \alpha \beta} p_{1}^{\mu} p_{2}^{\nu} p_{\perp}^{\alpha} \epsilon_{4}^{\beta}\left(F_{6}^{\lambda_{1} \lambda_{2}} p_{1} \cdot \epsilon_{3}+F_{7}^{\lambda_{1} \lambda_{2}} p_{2} \cdot \epsilon_{3}\right) \\
\left.+i \epsilon_{\mu \nu \alpha \beta} p_{1}^{\mu} p_{2}^{\nu} p_{\perp}^{\alpha} \epsilon_{3}^{\beta}\left(F_{8}^{\lambda_{1} \lambda_{2}} p_{1} \cdot \epsilon_{4}+F_{9}^{\lambda_{1} \lambda_{2}} p_{2} \cdot \epsilon_{4}\right)\right] .
\end{gathered}
$$

In eq. (2.16) $N_{\lambda_{1} \lambda_{2}}$ are the normalization factors for left-left and left-right polarization cases

$$
\mathcal{N}_{L L}=\frac{\langle 12\rangle}{[12] s}, \quad \mathcal{N}_{L R}=\frac{\left\langle 1 \hat{p}_{\perp} 2\right]}{\left[1 \hat{p}_{\perp} 2\right\rangle p_{\perp}^{2} s^{2}},
$$

and $F_{i=1 \ldots 9}^{\lambda_{1} \lambda_{2}}$ are helicity-dependent form factors that are functions of the Mandelstam variables and the invariant masses of vector bosons.

To account for transitions of vector bosons to final state leptons, their polarization vectors are replaced by matrix elements of vector and axial-vector currents. We therefore choose

$$
\epsilon_{3 L}^{\mu}=\left\langle 5\left|\gamma^{\mu}\right| 6\right], \quad \epsilon_{3 R}^{\mu}=\left\langle 6\left|\gamma^{\mu}\right| 5\right], \quad \epsilon_{4 L}^{\mu}=\left\langle 7\left|\gamma^{\mu}\right| 8\right], \quad \epsilon_{4 R}^{\mu}=\left\langle 8\left|\gamma^{\mu}\right| 7\right] .
$$

We note that, although we need helicity amplitudes for all possible helicity combinations of leptons, it is sufficient to compute just one of them since other helicity amplitudes 

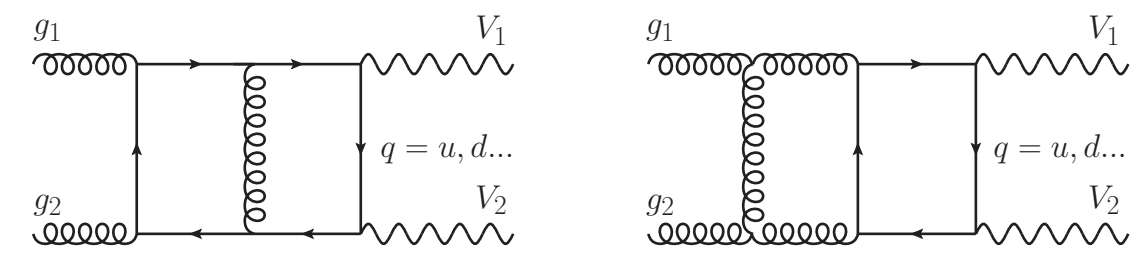

Figure 1. Representative two-loop diagrams that describe production of vector boson pairs in gluon fusion.

can be obtained using simple replacement rules. The amplitudes for left-handed polarization of both electroweak vector bosons read

$$
\begin{aligned}
\mathcal{A}_{3 L 4 L}^{\lambda_{1} \lambda_{2}}= & \mathcal{N}_{\lambda_{1} \lambda_{2}}\left\{\left(F_{1}^{\lambda_{1} \lambda_{2}}\langle 15\rangle[61]+F_{2}^{\lambda_{1} \lambda_{2}}\langle 25\rangle[62]\right)\langle 17\rangle[81]\right. \\
& +\left(F_{3}^{\lambda_{1} \lambda_{2}}\langle 15\rangle[61]+F_{4}^{\lambda_{1} \lambda_{2}}\langle 25\rangle[62]\right)\langle 27\rangle[82]+2 F_{5}^{\lambda_{1} \lambda_{2}}\langle 57\rangle[86] \\
& +\frac{1}{2}\left(F_{6}^{\lambda_{1} \lambda_{2}}\langle 15\rangle[61]+F_{7}^{\lambda_{1} \lambda_{2}}\langle 25\rangle[62]\right)(\langle 12\rangle\langle 78\rangle[81][82]+\langle 17\rangle\langle 27\rangle[21][87]) \\
& \left.-\frac{1}{2}\left(F_{8}^{\lambda_{1} \lambda_{2}}\langle 17\rangle[81]+F_{9}^{\lambda_{1} \lambda_{2}}\langle 27\rangle[82]\right)(\langle 12\rangle\langle 56\rangle[61][62]+\langle 15\rangle\langle 25\rangle[21][65])\right\} .
\end{aligned}
$$

Amplitudes for right-handed polarizations of the vector boson with momentum $p_{3}\left(p_{4}\right)$ are obtained from the above ones upon the replacement $5 \leftrightarrow 6(7 \leftrightarrow 8)$. Finally, all remaining helicity amplitudes can be obtain by replacing all angle brackets in spinor products with square brackets in eq. (2.19) and vice versa

$$
\mathcal{A}_{3 R 4 R}^{-\lambda_{1}-\lambda_{2}}=\mathcal{A}_{3 L 4 L}^{\lambda_{1} \lambda_{2}}[\langle i j\rangle \leftrightarrow[i j]]
$$

The $F$ form factors that enter the amplitudes are expressed through either $T$ or $G$ form factors. This can be done in a straightforward way using eqs. (2.7), (2.13), (2.15), (2.16). Examples of corresponding relations are given in the appendix.

\section{Calculation of the amplitude}

We apply the set-up described in the previous section to the calculation of gluon-fusion amplitude. There are 93 non-vanishing two-loop diagrams that contribute to the $g g \rightarrow V V$ amplitude; some examples are shown in figure 1. We generate the relevant diagrams using QGRAF [23] and process them with Maple and Form [24]. We compute the contribution of every diagram to the $G$ and eventually $F$ form factors. At this point, the result is expressed in terms of two-loop tensor integrals. These integrals can be classified in terms of six different topologies, three of which are planar and three are non-planar $[9,10]$. The tensor integrals are expressed through the master integrals computed in refs. $[9,10]$, using integration-by-parts technology $[21,22]$. We employ the program FIRE [25-27] to achieve this. Combining contributions of different diagrams, we obtain the results for the eighteen form factors (nine for $L L$ gluon helicity configuration and nine for $L R$ gluon 
helicity configuration) that are required to describe all helicity amplitudes for $g g \rightarrow V_{1} V_{2}$ process. We note that, compared to the calculation of $q \bar{q} \rightarrow V_{1} V_{2}$ amplitude, the case of $g g \rightarrow V_{1} V_{2}$ requires more complicated reduction since tensor integrals of a higher rank appear. Nevertheless, FIRE can successfully deal with this challenge.

As we already mentioned, the helicity amplitudes are expressed in terms of master integrals computed in refs. [9, 10]. The analytic expressions for these master integrals involve various functions, including logarithms, polylogarithms of multiple ranks as well as generalized Goncharov polylogarithms. To compute the latter, we use their numerical implementation [28] in the computer algebra program $\mathrm{GiNaC}$ [29]. We note that $\mathrm{GiNaC}$ can be called from both Mathematica and Fortran providing multiple options for the numerical evaluation of the amplitude.

The $g g \rightarrow V_{1} V_{2}$ amplitude appears for the first time at one loop; for this reason this amplitude is ultraviolet and infra-red finite. The two-loop $g g \rightarrow V_{1} V_{2}$ amplitude contains at most $\mathcal{O}\left(1 / \epsilon^{2}\right)$ singularities, where $\epsilon=(4-d) / 2$ is the parameter of dimensional regularization. The divergences of the two-loop $g g \rightarrow V_{1} V_{2}$ amplitude can be predicted in terms of the one-loop amplitude using results of ref. [30]. The relation between one- and two-loop amplitudes becomes very simple if expressed through bare, rather than renormalized QCD coupling. It reads

$$
\mathcal{A}_{2}=-\frac{C_{A}}{\epsilon^{2}} e^{\epsilon i \pi} \mathcal{A}_{1}+\mathcal{O}\left(\epsilon^{0}\right)
$$

where $C_{A}=3$ is the $\mathrm{QCD}$ color factor and $\mathcal{A}_{1,2}$ are defined through the following expression for the amplitude

$$
\mathcal{A}=\frac{a_{0}}{2}\left[s^{-\epsilon} \mathcal{A}_{1}+a_{0} s^{-2 \epsilon} \mathcal{A}_{2}+\mathcal{O}\left(\alpha_{s}^{2}\right)\right]
$$

In eq. (3.2) we use $a_{0}=\alpha_{s}^{(0)} \Gamma(1+\epsilon)(4 \pi)^{\epsilon} /(2 \pi)$, where $\alpha_{s}^{(0)}$ is the bare QCD coupling constant.

A connection between divergences of the two-loop amplitude $\mathcal{A}_{2}$ and the one-loop amplitude $\mathcal{A}_{1}$ given by eq. (3.1) is important for checks of the correctness of the calculation since the computation of $\mathcal{A}_{2}$ proceeds without separation into divergent and convergent parts until the very end.

We are now in position to present some numerical results for the $g g \rightarrow V_{1} V_{2}$ amplitude. To this end, we choose kinematics of an irreducible background to Higgs boson production and take the center-of-mass energy $\sqrt{s}$ to be the mass of the Higgs boson $\sqrt{s}=m_{H}=$ $125 \mathrm{GeV}$. The invariant mass of the vector boson $V_{1}$ is set to $p_{3}^{2}=m_{W}^{2}$, with $m_{W}=$ $80.419 \mathrm{GeV}$. The invariant mass of the second vector boson $V_{2}$ is set to $25 \mathrm{GeV}$. We take the vector boson scattering angle in the center-of-mass collision frame to be $\pi / 3$ radians. We also take decay angles of the lepton $l_{5}$ in the rest frame of the boson $V_{1}$ to be $\theta_{5}=\pi / 4$ and $\varphi_{5}=\pi / 2$ and decay angles of the lepton $l_{7}$ in the rest frame of the boson $V_{2}$ to be $\theta_{7}=\pi / 6$ and $\varphi_{7}=\pi$. The four-momenta of initial and final state particles in GeV are 


\begin{tabular}{|l|c|c|c|c|}
\hline Helicity & $\mathcal{A}_{1}(\epsilon=0)$ & $\mathcal{A}^{(2)} / \mathcal{A}_{1}(\epsilon=0), 1 / \epsilon^{2}$ & $\mathcal{A}^{(2)} / \mathcal{A}_{1}(\epsilon=0), 1 / \epsilon$ & $\mathcal{A}^{(2)} / \mathcal{A}_{1}(\epsilon=0), \epsilon^{0}$ \\
\hline LLLL & $-5169.9932+i 10017.414$ & -3.0 & $-9.45694415-i 16.4895884$ & $42.4852911-i 65.01495$ \\
\hline LRLL & $-6427.41534-i 2610.6160$ & -3.0 & $-14.9422361-i 9.2198662$ & $-6.774561932-i 71.66763$ \\
\hline
\end{tabular}

Table 1. Leading and next-to-leading order helicity amplitudes. Momenta of external particles are given in the main text of the paper.

given by

$$
\begin{aligned}
& p_{1}=(62.5,0,0,62.5), \quad p_{2}=(62.5,0,0,-62.5), \\
& p_{5}=(48.2561024468725,13.8697156788798,-28.4324101181205,36.4400941989053), \\
& p_{6}=(37.6127597971275,12.2010429705974,28.4324101181205,-21.3881346746519), \\
& p_{7}=(19.5655688780000,-19.2853793247386,0,3.29933778517879), \\
& p_{8}=(19.5655688780000,-6.78537932473856,0,-18.3512973094322) .
\end{aligned}
$$

Our results for leading and next-to-leading order helicity amplitudes at the kinematic point eq. (3.3) are shown in table 1. The divergent terms of the next-to-leading order amplitude are compared to predictions based on eq. (3.1) and perfect agreement is found. We also compared the leading order helicity amplitude with the results of previous computations [31-34], as implemented in the program MCFM [35], and found agreement. Finally, we note that we compared the numerical results for helicity amplitudes reported in table 1 with the results of the independent calculation [36] and found complete agreement.

Finally, it is interesting to explore the numerical stability of $g g \rightarrow V_{1} V_{2}$ amplitudes that we computed in this paper. The numerical stability of such amplitudes is known to be a potentially sensitive issue as earlier one-loop studies showed, see e.g. refs. [34, 37]. To study numerical stability, we consider the same kinematic point as described above but we treat the vector boson scattering angle as a free parameter. We then compare the results of the double-precision implementation of the form factors in a Fortran program with, effectively, arbitrary-precision calculation in Mathematica. We find that helicity amplitudes computed in these two different ways agree well for values of the scattering angle as small (large) as $\theta=1(\theta=179)$ degrees. For such angles, the transverse momentum of a vector boson is just $0.5 \mathrm{GeV}$. We therefore conclude that our numerical implementation of helicity amplitudes is sufficiently stable to allow their use in realistic numerical calculations. As a further illustration of these numerical results, in figure 2 we show absolute values of helicity amplitudes as a function of the scattering angle.

\section{Conclusions}

In this paper, we computed the helicity amplitudes for the production of electroweak gauge bosons in gluon fusion $g g \rightarrow V_{1} V_{2}$, mediated by massless quark loops. The electroweak gauge bosons are allowed to have different masses and be off-shell; their decays to fermion pairs are taken into account explicitly. The helicity amplitudes for $g g \rightarrow V_{1} V_{2}$ are described by nine helicity-dependent form factors. We construct projection operators to compute those form factors from Feynman diagrams, using integration-by-parts identities and the 


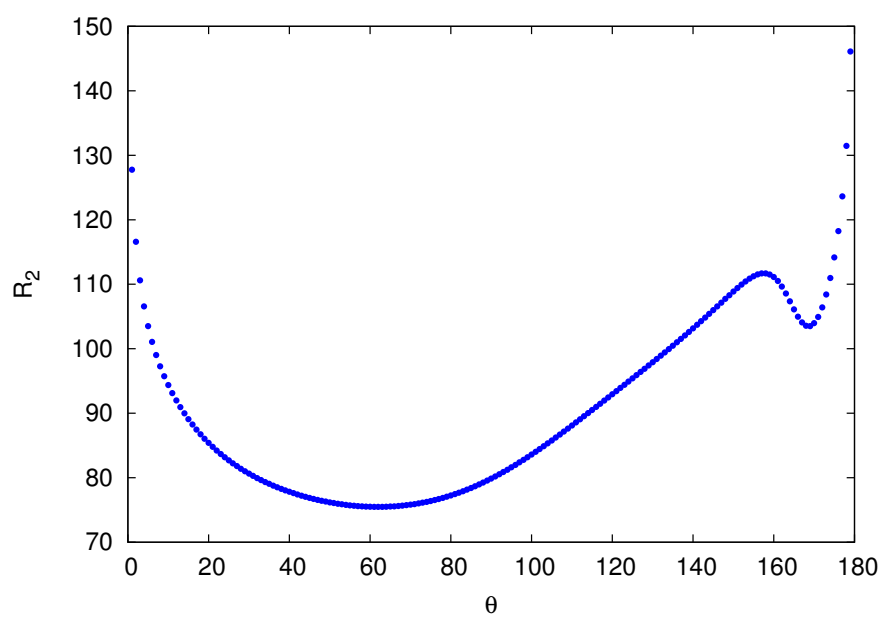

Figure 2. The ratio of finite parts of two- and one-loop helicity amplitudes $R_{2}=\mid A_{2}(L L)+$ $A_{2}(L R) \mid /\left(\left|A_{1}(L L)\right|+\left|A_{1}(L R)\right|\right)$ as a function of the vector-boson scattering angle.

master integrals calculated by us previously. Analytic results for helicity amplitudes are implemented in a Fortran code that is available from the authors upon request.

The results for the scattering amplitudes $g g \rightarrow W^{+} W^{-}$and $g g \rightarrow Z Z$ obtained in this paper open up an opportunity to compute the NLO QCD corrections to the production of a pair of electroweak vector boson in gluon fusion. Such calculations are interesting both at low and high center-of-mass collision energies. In the former case, they allow for a more accurate estimate of the irreducible background to Higgs production and the possible signal/background interference effects. In the latter case, precise predictions for $g g \rightarrow Z Z$ are important for improving prospects of constraining total Higgs boson decay width following refs. [38-40], as well as for more generic Higgs off-shell studies [41]. The corresponding real emission corrections $g g \rightarrow Z Z g$ have to be calculated in this case. Such calculations are clearly possible with the existing one-loop technology [42-45] and will be done in the near future.

\section{Acknowledgments}

K.M. is grateful to L. Tancredi for useful conversations. F.C. would like to thank the Institute for Advanced Studies, Princeton, for the hospitality extended to him during completion of this paper. We are grateful to A. von Manteuffel and L. Tancredi for the opportunity to compare results for $g g \rightarrow V_{1} V_{2}$ amplitude prior to their publication. J.M.H. is supported in part by the DOE grant DE-SC0009988 and by the Marvin L. Goldberger fund. The research of K.M. was supported by Karlsruhe Institute of Technology through its startup grant. A.S. is supported in part by DFG through SFB/TR 9. V.S. is supported in part by the Alexander von Humboldt Foundation (Humboldt Forschungspreis). 


\section{A The relations between various form factors}

For the two possible helicity combinations, the invariant form factors, expressed through $T$ form-factors, read

$$
\begin{aligned}
& F_{1}^{L L}=\frac{1}{2}\left[\left(m_{3}^{2}-u\right)\left(T_{12}+T_{8}\right)-\left(m_{4}^{2}-t\right)\left(T_{10}+T_{14}\right)-s\left(p_{\perp}^{2} T_{17}+2 T_{4}\right)\right], \\
& F_{2}^{L L}=\frac{1}{2}\left[\left(m_{3}^{2}-t\right)\left(T_{12}+T_{8}\right)-\left(m_{4}^{2}-t\right)\left(T_{11}+T_{15}\right)-p_{\perp}^{2} s T_{19}-2 s T_{6}+2 T_{2}+2 T_{3}\right], \\
& F_{3}^{L L}=\frac{1}{2}\left[\left(m_{3}^{2}-u\right)\left(T_{13}+T_{9}\right)-\left(m_{4}^{2}-u\right)\left(T_{10}+T_{14}\right)-p_{\perp}^{2} s T_{18}-2 s T_{5}+2 T_{2}+2 T_{3}\right], \\
& F_{4}^{L L}=\frac{1}{2}\left[\left(m_{3}^{2}-t\right)\left(T_{13}+T_{9}\right)-\left(m_{4}^{2}-u\right)\left(T_{11}+T_{15}\right)-s\left(p_{\perp}^{2} T_{20}+2 T_{7}\right)\right], \\
& F_{5}^{L L}=-\frac{1}{2} s\left(p_{\perp}^{2} T_{16}+2 T_{1}+T_{2}+T_{3}\right) \\
& F_{6}^{L L}=\frac{\left(m_{3}^{2}-u\right)\left(T_{3}-T_{2}\right)}{p_{\perp}^{2} s}-T_{10}+T_{14}, \\
& F_{7}^{L L}=\frac{\left(m_{3}^{2}-t\right)\left(T_{3}-T_{2}\right)}{p_{\perp}^{2} s}-T_{11}+T_{15}, \\
& F_{8}^{L L}=\frac{\left(m_{4}^{2}-t\right)\left(T_{3}-T_{2}\right)}{p_{\perp}^{2} s}+T_{12}-T_{8}, \\
& F_{9}^{L L}=\frac{\left(m_{4}^{2}-u\right)\left(T_{3}-T_{2}\right)}{p_{\perp}^{2} s}+T_{13}-T_{9} ; \\
& F_{1}^{L R}=\frac{1}{2}\left[\left(m_{4}^{2}-t\right)\left(p_{\perp}^{2} s\left(T_{10}+T_{14}\right)-2\left(m_{3}^{2}-u\right)\left(T_{2}+T_{3}\right)\right)\right. \\
& \left.+p_{\perp}^{2} s\left(p_{\perp}^{2} s T_{17}-\left(m_{3}^{2}-u\right)\left(T_{12}+T_{8}\right)\right)\right], \\
& F_{2}^{L R}=\frac{1}{2}\left[\left(m_{4}^{2}-t\right)\left(p_{\perp}^{2} s\left(T_{11}+T_{15}\right)-2\left(m_{3}^{2}-t\right)\left(T_{2}+T_{3}\right)\right)\right. \\
& \left.-p_{\perp}^{2} s\left(\left(m_{3}^{2}-t\right)\left(T_{12}+T_{8}\right)-p_{\perp}^{2} s T_{19}-2\left(T_{2}+T_{3}\right)\right)\right], \\
& F_{3}^{L R}=\frac{1}{2} p_{\perp}^{2} s\left(-\left(m_{3}^{2}-u\right)\left(T_{13}+T_{9}\right)+\left(m_{4}^{2}-u\right)\left(T_{10}+T_{14}\right)+p_{\perp}^{2} s T_{18}\right) \\
& +\left(T_{2}+T_{3}\right)\left(p_{\perp}^{2} s-\left(m_{3}^{2}-u\right)\left(m_{4}^{2}-u\right)\right), \\
& F_{4}^{L R}=\frac{1}{2}\left(\left(m_{4}^{2}-u\right)\left(p_{\perp}^{2} s\left(T_{11}+T_{15}\right)-2\left(m_{3}^{2}-t\right)\left(T_{2}+T_{3}\right)\right)\right. \\
& \left.+p_{\perp}^{2} s\left(p_{\perp}^{2} s T_{20}-\left(m_{3}^{2}-t\right)\left(T_{13}+T_{9}\right)\right)\right), \\
& F_{5}^{L R}=\frac{1}{2} p_{\perp}^{2} s^{2}\left(p_{\perp}^{2} T_{16}-T_{2}-T_{3}\right), \\
& F_{6}^{L R}=p_{\perp}^{2} s\left(T_{10}+T_{14}\right)-\left(m_{3}^{2}-u\right)\left(T_{2}+T_{3}\right), \\
& F_{7}^{L R}=p_{\perp}^{2} s\left(T_{11}+T_{15}\right)-\left(m_{3}^{2}-t\right)\left(T_{2}+T_{3}\right), \\
& F_{8}^{L R}=\left(m_{4}^{2}-t\right)\left(T_{2}+T_{3}\right)+p_{\perp}^{2} s\left(T_{12}+T_{8}\right), \\
& F_{9}^{L R}=\left(m_{4}^{2}-u\right)\left(T_{2}+T_{3}\right)+p_{\perp}^{2} s\left(T_{13}+T_{9}\right) .
\end{aligned}
$$


The square of the transverse momentum written through Mandelstam invariants reads $p_{\perp}^{2}=-\left(t u-m_{3}^{2} m_{4}^{2}\right) / s$.

When the $F$ form factors are expressed in terms of projections $G_{1, \ldots, 20}$ shown earlier, the results appear to be relatively simple. In particular, all spurious poles in $d-4$, present in the relations between $T$ 's and $G$ 's cancel out in the relations between $F$ 's and $G$ 's. To give an example of these relations, we show results for a few of the simplest form factors for the $L L$ amplitude

$$
\begin{aligned}
& F_{6}^{L L}=\frac{\left(m_{3}^{2}-u\right)\left(G_{12}+G_{16}\right)}{(d-3)\left(m_{3}^{2}-t\right)}+\frac{s\left(m_{3}^{2}+p_{\perp}^{2}\right)\left(G_{13}+G_{17}\right)}{(d-3)\left(m_{3}^{2}-t\right)}, \\
& F_{7}^{L L}=\frac{G_{12}+G_{16}+\left(t-m_{3}^{2}\right)\left(G_{13}+G_{17}\right)}{d-3}, \\
& F_{8}^{L L}=\frac{\left(m_{4}^{2}-t\right) G_{10}-s\left(m_{4}^{2}+p_{\perp}^{2}\right) G_{11}}{(d-3)\left(m_{4}^{2}-u\right)}+\frac{G_{14}+\left(m_{4}^{2}-t\right) G_{15}}{d-3}, \\
& F_{9}^{L L}=\frac{\left(u-m_{4}^{2}\right) G_{11}-G_{10}}{d-3}+\frac{\left(u-m_{4}^{2}\right) G_{14}+s\left(m_{4}^{2}+p_{\perp}^{2}\right) G_{15}}{(d-3)\left(m_{4}^{2}-t\right)} .
\end{aligned}
$$

Open Access. This article is distributed under the terms of the Creative Commons Attribution License (CC-BY 4.0), which permits any use, distribution and reproduction in any medium, provided the original author(s) and source are credited.

\section{References}

[1] F. Cascioli et al., ZZ production at hadron colliders in NNLO QCD, Phys. Lett. B 735 (2014) 311 [arXiv: 1405.2219] [INSPIRE].

[2] T. Gehrmann et al., $W^{+} W^{-}$production at hadron colliders in next to next to leading order QCD, Phys. Rev. Lett. 113 (2014) 212001 [arXiv:1408.5243] [INSPIRE].

[3] F. Cascioli et al., Precise Higgs-background predictions: merging NLO QCD and squared quark-loop corrections to four-lepton $+0,1$ jet production, JHEP 01 (2014) 046 [arXiv: 1309.0500] [INSPIRE].

[4] T. Binoth, M. Ciccolini, N. Kauer and M. Krämer, Gluon-induced W-boson pair production at the LHC, JHEP 12 (2006) 046 [hep-ph/0611170] [INSPIRE].

[5] M. Spira, A. Djouadi, D. Graudenz and P.M. Zerwas, Higgs boson production at the LHC, Nucl. Phys. B 453 (1995) 17 [hep-ph/9504378] [InSPIRE].

[6] S. Dawson, S. Dittmaier and M. Spira, Neutral Higgs boson pair production at hadron colliders: QCD corrections, Phys. Rev. D 58 (1998) 115012 [hep-ph/9805244] [INSPIRE].

[7] L. Altenkamp, S. Dittmaier, R.V. Harlander, H. Rzehak and T.J.E. Zirke, Gluon-induced Higgs-strahlung at next-to-leading order QCD, JHEP 02 (2013) 078 [arXiv:1211.5015] [INSPIRE].

[8] Z. Bern, L.J. Dixon and C. Schmidt, Isolating a light Higgs boson from the diphoton background at the CERN LHC, Phys. Rev. D 66 (2002) 074018 [hep-ph/0206194] [InSPIRE].

[9] J.M. Henn, K. Melnikov and V.A. Smirnov, Two-loop planar master integrals for the production of off-shell vector bosons in hadron collisions, JHEP 05 (2014) 090 [arXiv: 1402 .7078] [INSPIRE]. 
[10] F. Caola, J.M. Henn, K. Melnikov and V.A. Smirnov, Non-planar master integrals for the production of two off-shell vector bosons in collisions of massless partons, JHEP 09 (2014) 043 [arXiv: 1404.5590] [INSPIRE].

[11] C.G. Papadopoulos, D. Tommasini and C. Wever, Two-loop master integrals with the simplified differential equations approach, JHEP 01 (2015) 072 [arXiv: 1409.6114] [INSPIRE].

[12] T. Gehrmann, A. von Manteuffel and L. Tancredi, The two-loop helicity amplitudes for $q \bar{q}^{\prime} \rightarrow V_{1} V_{2} \rightarrow 4$ leptons, arXiv:1503.04812 [INSPIRE].

[13] T. Gehrmann, A. von Manteuffel, L. Tancredi and E. Weihs, The two-loop master integrals for $q \bar{q} \rightarrow V V$, JHEP 06 (2014) 032 [arXiv: 1404.4853] [INSPIRE].

[14] T. Gehrmann, L. Tancredi and E. Weihs, Two-loop master integrals for $q \bar{q} \rightarrow V V$ : the planar topologies, JHEP 08 (2013) 070 [arXiv: 1306.6344] [INSPIRE].

[15] J.M. Campbell, R.K. Ellis and C. Williams, Gluon-gluon contributions to $W^{+} W^{-}$production and Higgs interference effects, JHEP 10 (2011) 005 [arXiv:1107.5569] [INSPIRE].

[16] K. Melnikov and M. Dowling, Production of two Z-bosons in gluon fusion in the heavy top quark approximation, Phys. Lett. B 744 (2015) 43 [arXiv:1503.01274] [INSPIRE].

[17] F. Caola, J.M. Henn, K. Melnikov, A.V. Smirnov and V.A. Smirnov, Two-loop helicity amplitudes for the production of two off-shell electroweak bosons in quark-antiquark collisions, JHEP 11 (2014) 041 [arXiv: 1408.6409] [INSPIRE].

[18] K. Nishijima, Generalized Furry's theorem for closed loops, Prog. Theor. Phys. 6 (1951) 614.

[19] E.W.N. Glover and J.J. van der Bij, Vector boson pair production via gluon fusion, Phys. Lett. B 219 (1989) 488 [INSPIRE].

[20] C. Kao and D.A. Dicus, Production of $W^{+} W^{-}$from gluon fusion, Phys. Rev. D 43 (1991) 1555 [INSPIRE].

[21] F.V. Tkachov, A theorem on analytical calculability of four loop renormalization group functions, Phys. Lett. B 100 (1981) 65 [INSPIRE].

[22] K.G. Chetyrkin and F.V. Tkachov, Integration by parts: the algorithm to calculate $\beta$-functions in 4 loops, Nucl. Phys. B 192 (1981) 159 [INSPIRE].

[23] P. Nogueira, Automatic Feynman graph generation, J. Comput. Phys. 105 (1993) 279 [INSPIRE].

[24] J. Kuipers, T. Ueda, J.A.M. Vermaseren and J. Vollinga, FORM version 4.0, Comput. Phys. Commun. 184 (2013) 1453 [arXiv: 1203.6543] [INSPIRE].

[25] A.V. Smirnov, Algorithm FIRE - Feynman Integral REduction, JHEP 10 (2008) 107 [arXiv:0807.3243] [INSPIRE].

[26] A.V. Smirnov and V.A. Smirnov, FIRE4, LiteRed and accompanying tools to solve integration by parts relations, Comput. Phys. Commun. 184 (2013) 2820 [arXiv:1302.5885] [INSPIRE].

[27] A.V. Smirnov, FIRE5: a C++ implementation of Feynman Integral REduction, Comput. Phys. Commun. 189 (2014) 182 [arXiv:1408.2372] [INSPIRE].

[28] J. Vollinga and S. Weinzierl, Numerical evaluation of multiple polylogarithms, Comput. Phys. Commun. 167 (2005) 177 [hep-ph/0410259] [INSPIRE].

[29] C. W. Bauer, A. Frink and R. Kreckel, Introduction to the GiNaC framework for symbolic computation within the C++ programming language, J. Symb. Comput. 33 (2002) 1 [cs/0004015].

[30] S. Catani, The singular behavior of QCD amplitudes at two loop order, Phys. Lett. B 427 (1998) 161 [hep-ph/9802439] [inSPIRE]. 
[31] E.W.N. Glover and J.J. van der Bij, Z boson pair production via gluon fusion, Nucl. Phys. B 321 (1989) 561 [INSPIRE].

[32] T. Matsuura and J.J. van der Bij, Characteristics of leptonic signals for $Z$ boson pairs at hadron colliders, Z. Phys. C 51 (1991) 259 [INSPIRE].

[33] C. Zecher, T. Matsuura and J.J. van der Bij, Leptonic signals from off-shell Z boson pairs at hadron colliders, Z. Phys. C 64 (1994) 219 [hep-ph/9404295] [INSPIRE].

[34] T. Binoth, M. Ciccolini, N. Kauer and M. Krämer, Gluon-induced WW background to Higgs boson searches at the LHC, JHEP 03 (2005) 065 [hep-ph/0503094] [INSPIRE].

[35] J.M. Campbell, R.K. Ellis and C. Williams, http://mcfm.fnal.gov.

[36] A. von Manteuffel and L. Tancredi, The two-loop helicity amplitudes for $g g \rightarrow V_{1} V_{2} \rightarrow 4$ leptons, arXiv:1503.08835 [INSPIRE].

[37] J.M. Campbell, R.K. Ellis and C. Williams, Bounding the Higgs width at the LHC using full analytic results for $g g \rightarrow e^{-} e^{+} \mu^{-} \mu^{+}$, JHEP 04 (2014) 060 [arXiv: 1311.3589] [INSPIRE].

[38] F. Caola and K. Melnikov, Constraining the Higgs boson width with $Z Z$ production at the LHC, Phys. Rev. D 88 (2013) 054024 [arXiv:1307.4935] [INSPIRE].

[39] CMS collaboration, Constraints on the Higgs boson width from off-shell production and decay to Z-boson pairs, Phys. Lett. B 736 (2014) 64 [arXiv:1405.3455] [INSPIRE].

[40] ATLAS collaboration, Determination of the off-shell Higgs boson signal strength in the high-mass ZZ final state with the ATLAS detector, ATLAS-CONF-2014-042 (2014) [ATLAS-COM-CONF-2014-052].

[41] N. Kauer and G. Passarino, Inadequacy of zero-width approximation for a light Higgs boson signal, JHEP 08 (2012) 116 [arXiv: 1206.4803] [INSPIRE].

[42] J.M. Campbell, R.K. Ellis, E. Furlan and R. Röntsch, Interference effects for Higgs boson mediated Z-pair plus jet production, Phys. Rev. D 90 (2014) 093008 [arXiv:1409.1897] [INSPIRE].

[43] T. Melia, K. Melnikov, R. Rontsch, M. Schulze and G. Zanderighi, Gluon fusion contribution to $W^{+} W^{-}+$jet production, JHEP 08 (2012) 115 [arXiv:1205.6987] [INSPIRE].

[44] P. Agrawal and A. Shivaji, Di-vector boson + jet production via gluon fusion at hadron colliders, Phys. Rev. D 86 (2012) 073013 [arXiv:1207.2927] [InSPIRE].

[45] F. Campanario, Q. Li, M. Rauch and M. Spira, $Z Z+$ jet production via gluon fusion at the LHC, JHEP 06 (2013) 069 [arXiv:1211.5429] [INSPIRE]. 